DOI $10.31489 / 2020 \mathrm{M} 3 / 18-25$

MSC 35P15

Karwan H.F. Jwamer ${ }^{1}$ and Rando R. Q. Rasul ${ }^{2}$

${ }^{1}$ Mathematics Department, College of Science, University of Sulaimani, Sulaimani, Kurdistan Riegion of Iraq

${ }^{2}$ Department of Mathematics, School of basic Education, University of Sulaimani, Kurdistan region, Iraq

(E-mail:karwan.jwamer@univsul.edu.iq,rando.qadir@univsul.edu.iq)

\title{
A comparison between the fourth order linear differential equation with its boundary value problem
}

\begin{abstract}
In this paper, we study a fourth order linear differential equation. We found an upper bound for the solutions of this differential equation and also, we prove that all the solutions are in $L^{4}(0, \infty)$. By comparing these results we obtain that all the eigenfunction of the boundary value problem generated by this differential equation are bounded and in $L^{4}(0, \infty)$.

Keywords: linear differential equation, eigenvalue, eigenfunction, upper bound, linearly independent solution, $L^{2}(0, \infty)$, wrongskian, Gronwall inequality, Variation of parameters.
\end{abstract}

\section{Introduction}

The method of finding an upper bound for the solutions of a differential equation has been investigated by many authors. In papers $[2,4]$ by authors were investigated the solutions of the second

order linear differential equation. They obtained some important properties of this equation such that all solutions of the differential equation are bounded and in the space $L^{2}(0, \infty)$. Here $L^{2}(0, \infty)$ is the space of all functions $f$ which are continuous and satisfy the conditions:

$$
\int_{0}^{\infty}|f(x)|^{2} d x<\infty
$$

The estimate of upper bounds for the eigenfunctions of a boundary value problem was investigated by many authors. In papers $[2-6,10]$ by authors were investigated a second order differential equation of the form

$$
y^{\prime \prime}+q(x) y=\lambda^{2} \rho(x) y, x \in[0, a] .
$$

They found a normalized eigenfunctions for this problem and an upper bound for this solution under a certain condions.

Methods of finding of general solution of a fourth order differential equation were studied by many authors, see: $[1,7-9,11]$.

This paper is specified to study some important properties of solutions of a fourth order linear differential equation of the form:

$$
y^{(4)}(x)+\{q(x)+r(x)\} y(x)=0, \quad 0 \leq x<\infty,
$$

where $r(x)$ is a function satisfying the condition:

$$
\int_{0}^{\infty}|r(x)| d x<\infty
$$

We investigate whether the solutions of (1) are related to any general properties such as boundedness of the solutions of the differential equation

$$
y^{(4)}(x)+q(x) y(x)=0, \quad 0 \leq x<\infty .
$$


Let $L^{4}(0, \infty)$ is the space of all continuous functions $f$ for which satisfy the condition

$$
\int_{0}^{\infty}|f(x)|^{4} d x<\infty
$$

In this paper we show that all solutions of (1) are in $L^{4}(0, \infty)$. It is based on the fact that the solutions of $(3)$ are in $L^{4}(0, \infty)$ under the condition (2). Moreover, we show that eigenfunctions of the boundary value problem which is generated by the differential equation $y^{(4)}(x)+\{\lambda+r(x)\} y(x)=0$ are bounded under a certain condion.

Let $f(x)$ and $g(x)$ be real-valued, continuous, and nonnegative in $[a, b]$ and suppose that $f(x) \leq c+\int_{a}^{x} f(t) g(t) d t$, in $[a, b]$ where $c>0$ is a constant. Then,

$$
f(x) \leq c e^{\int_{a}^{x} g(t) d t} .
$$

This is known as Gronwall inequality [2].

\section{Expression for the solutions}

In this section we found a general solutions for (1) by using the method of variation of parameter. We need some properties of the differential equations (1) and (3) which are immediate consequence of the results of chapter two in [2].

Lemma 1. There are solutions $\phi_{j}(x),\{j=1,2,3,4\}$ of (3) such that $W\left(\phi_{1}, \phi_{3}, \phi_{2}, \phi_{4}\right)=1$ in $[0, \infty)$.

Proof. Let $y_{1}(x), y_{2}(x), y_{3}(x)$ and $y_{4}(x)$ be a fundamental system of solution of (3), then we obtain $W\left(\phi_{1}, \phi_{3}, \phi_{2}, \phi_{4}\right)=c$ in $[0, \infty)$, where $c$ is a non zero constant, we take $\phi_{1}(x)=y_{1}(x)$, $\phi_{2}(x)=y_{2}(x), \quad \phi_{3}(x)=y_{3}(x)$ and $\phi_{4}(x)=\frac{y_{4}(x)}{c}$, then we can easily establish that $W\left(\phi_{1}, \phi_{3}, \phi_{2}, \phi_{4}\right)=1$.

Lemma 2. If $\phi_{j}(x),\{j=1,2,3,4\}$ are as in Lemma 1 and $\psi(x)$ is any solution of (1), then there are unique constants $c_{j}$ for $j=1: 4$ such that

$$
\psi(x)=c_{1} \phi_{1}(x)+c_{2} \phi_{2}(x)+c_{3} \phi_{3}(x)+c_{4} \phi_{4}(x)+\psi_{0}(x),
$$

where

$$
\begin{aligned}
\psi_{0}(x)= & \int_{0}^{x}\left[\phi_{2}(t) \phi_{3}^{\prime}(t) \phi_{4}^{\prime \prime}(t) \phi_{1}(x)+\phi_{2}^{\prime \prime}(t) \phi_{3}(t) \phi_{4}^{\prime}(t) \phi_{1}(x)+\phi_{2}^{\prime}(t) \phi_{3}^{\prime \prime}(t) \phi_{4}(t) \phi_{1}(x)\right. \\
& -\phi_{2}^{\prime}(t) \phi_{3}(t) \phi_{4}^{\prime \prime}(t) \phi_{1}(x)-\phi_{2}(t) \phi_{3}^{\prime \prime}(t) \phi_{4}^{\prime}(t) \phi_{1}(x)-\phi_{2}^{\prime \prime}(t) \phi_{3}^{\prime}(t) \phi_{4}(t) \phi_{1}(x) \\
& -\phi_{1}(t) \phi_{3}^{\prime}(t) \phi_{4}^{\prime \prime}(t) \phi_{2}(x)-\phi_{1}^{\prime \prime}(t) \phi_{3}(t) \phi_{4}^{\prime}(t) \phi_{2}(x)-\phi_{1}^{\prime}(t) \phi_{3}^{\prime \prime}(t) \phi_{4}(t) \phi_{2}(x) \\
& +\phi_{1}^{\prime}(t) \phi_{3}(t) \phi_{4}^{\prime \prime}(t) \phi_{2}(x)+\phi_{1}(t) \phi_{3}^{\prime \prime}(t) \phi_{4}^{\prime}(t) \phi_{2}(x)+\phi_{1}^{\prime \prime}(t) \phi_{3}^{\prime}(t) \phi_{4}(t) \phi_{2}(x) \\
& +\phi_{1}(t) \phi_{2}^{\prime}(t) \phi_{4}^{\prime \prime}(t) \phi_{3}(x)+\phi_{1}^{\prime \prime}(t) \phi_{2}(t) \phi_{4}^{\prime}(t) \phi_{3}(x)+\phi_{1}^{\prime}(t) \phi_{2}^{\prime \prime}(t) \phi_{4}(t) \phi_{3}(x) \\
& -\phi_{1}^{\prime}(t) \phi_{2}(t) \phi_{4}^{\prime \prime}(t) \phi_{3}(x)-\phi_{1}(t) \phi_{2}^{\prime \prime}(t) \phi_{4}^{\prime}(t) \phi_{3}(x)-\phi_{1}^{\prime \prime}(t) \phi_{2}^{\prime}(t) \phi_{4}(t) \phi_{3}(x) \\
& -\phi_{1}(t) \phi_{2}^{\prime}(t) \phi_{3}^{\prime \prime}(t) \phi_{4}(x)-\phi_{1}^{\prime \prime}(t) \phi_{2}(t) \phi_{3}^{\prime}(t) \phi_{4}(x)-\phi_{1}^{\prime}(t) \phi_{2}^{\prime \prime}(t) \phi_{3}(t) \phi_{4}(x) \\
& \left.+\phi_{1}^{\prime}(t) \phi_{2}(t) \phi_{3}^{\prime \prime}(t) \phi_{4}(x)+\phi_{1}(t) \phi_{2}^{\prime \prime}(t) \phi_{3}^{\prime}(t) \phi_{4}(x)+\phi_{1}^{\prime \prime}(t) \phi_{2}^{\prime}(t) \phi_{3}(t) \phi_{4}(x)\right] \\
& \times r(t) \psi(t) d t .
\end{aligned}
$$

Proof. If $\psi(x)$ is a solution of (1), then as we see in [2] by using variation of parameter there is unique constants $c_{j}$ such that

$$
\psi(x)=c_{1} \phi_{1}(x)+c_{2} \phi_{2}(x)+c_{3} \phi_{3}(x)+c_{4} \phi_{4}(x)+\psi_{0}(x),
$$

where

$$
\psi_{0}(x)=c_{1}(x) \phi_{1}(x)+c_{2}(x) \phi_{2}(x)+c_{3}(x) \phi_{3}(x)+c_{4}(x) \phi_{4}(x)
$$


and

$$
c_{r}(x)=\int_{0}^{x} \frac{W_{r}\left(\phi_{1}, \phi_{3}, \phi_{2}, \phi_{4}\right)(t)}{W\left(\phi_{1}, \phi_{3}, \phi_{2}, \phi_{4}\right)(t)} r(t) \psi(t) d t .
$$

From Lemma 1 it follows that $W\left(\phi_{1}, \phi_{3}, \phi_{2}, \phi_{4}\right)=1$.Therefore, (8) has the form

$$
c_{r}(x)=\int_{0}^{x} W_{r}\left(\phi_{1}, \phi_{3}, \phi_{2}, \phi_{4}\right)(t) r(t) \psi(t) d t .
$$

For $r=1$, we have that

$$
\begin{aligned}
& W_{1}\left(\phi_{1}, \phi_{2}, \phi_{3}, \phi_{4}\right)(t)=\left|\begin{array}{cccc}
0 & \phi_{2}(t) & \phi_{3}(t) & \phi_{4}(t) \\
0 & \phi_{2}^{\prime}(t) & \phi_{3}^{\prime}(t) & \phi_{4}^{\prime}(t) \\
0 & \phi_{2}^{\prime \prime}(t) & \phi_{3}^{\prime \prime}(t) & \phi_{4}^{\prime \prime}(t) \\
1 & \phi_{2}^{\prime \prime \prime}(t) & \phi_{3}^{\prime \prime \prime}(t) & \phi_{4}^{\prime \prime \prime}(t)
\end{array}\right| \\
& =\phi_{2}(t) \phi_{3}^{\prime}(t) \phi_{4}^{\prime \prime}(t)+\phi_{2}^{\prime \prime}(t) \phi_{3}(t) \phi_{4}^{\prime}(t)+\phi_{2}^{\prime}(t) \phi_{3}^{\prime \prime}(t) \phi_{4}(t)-\phi_{2}^{\prime}(t) \phi_{3}(t) \phi_{4}^{\prime \prime}(t) \\
& -\phi_{2}(t) \phi_{3}^{\prime \prime}(t) \phi_{4}^{\prime}(t)-\phi_{2}^{\prime \prime}(t) \phi_{3}^{\prime}(t) \phi_{4}(t) \text {. }
\end{aligned}
$$

That is

$$
\begin{aligned}
W_{1}\left(\phi_{1}, \phi_{2}, \phi_{3}, \phi_{4}\right)(t)= & \phi_{2}(t) \phi_{3}^{\prime}(t) \phi_{4}^{\prime \prime}(t)+\phi_{2}^{\prime \prime}(t) \phi_{3}(t) \phi_{4}^{\prime}(t)+\phi_{2}^{\prime}(t) \phi_{3}^{\prime \prime}(t) \phi_{4}(t) \\
& -\phi_{2}^{\prime}(t) \phi_{3}(t) \phi_{4}^{\prime \prime}(t)-\phi_{2}(t) \phi_{3}^{\prime \prime}(t) \phi_{4}^{\prime}(t)-\phi_{2}^{\prime \prime}(t) \phi_{3}^{\prime}(t) \phi_{4}(t) .
\end{aligned}
$$

For $r=2,3,4$,applying the same way, we obtain

$$
\begin{aligned}
W_{2}\left(\phi_{1}, \phi_{2}, \phi_{3}, \phi_{4}\right)(t)= & -\phi_{1}(t) \phi_{3}^{\prime}(t) \phi_{4}^{\prime \prime}(t)-\phi_{1}^{\prime \prime}(t) \phi_{3}(t) \phi_{4}^{\prime}(t)-\phi_{1}^{\prime}(t) \phi_{3}^{\prime \prime}(t) \phi_{4}(t) \\
& +\phi_{1}^{\prime}(t) \phi_{3}(t) \phi_{4}^{\prime \prime}(t)+\phi_{1}(t) \phi_{3}^{\prime \prime}(t) \phi_{4}^{\prime}(t)+\phi_{1}^{\prime \prime}(t) \phi_{3}^{\prime}(t) \phi_{4}(t), \\
W_{3}\left(\phi_{1}, \phi_{2}, \phi_{3}, \phi_{4}\right)(t)= & \phi_{1}(t) \phi_{2}^{\prime}(t) \phi_{4}^{\prime \prime}(t)+\phi_{1}^{\prime \prime}(t) \phi_{2}(t) \phi_{4}^{\prime}(t)+\phi_{1}^{\prime}(t) \phi_{2}^{\prime \prime}(t) \phi_{4}(t) \\
& -\phi_{1}^{\prime}(t) \phi_{2}(t) \phi_{4}^{\prime \prime}(t)-\phi_{1}(t) \phi_{2}^{\prime \prime}(t) \phi_{4}^{\prime}(t)-\phi_{1}^{\prime \prime}(t) \phi_{2}^{\prime}(t) \phi_{4}(t), \\
W_{4}\left(\phi_{1}, \phi_{2}, \phi_{3}, \phi_{4}\right)(t)= & -\phi_{1}(t) \phi_{2}^{\prime}(t) \phi_{3}^{\prime \prime}(t)-\phi_{1}^{\prime \prime}(t) \phi_{2}(t) \phi_{3}^{\prime}(t)-\phi_{1}^{\prime}(t) \phi_{2}^{\prime \prime}(t) \phi_{3}(t) \\
& +\phi_{1}^{\prime}(t) \phi_{2}(t) \phi_{3}^{\prime \prime}(t)+\phi_{1}(t) \phi_{2}^{\prime \prime}(t) \phi_{3}^{\prime}(t)+\phi_{1}^{\prime \prime}(t) \phi_{2}^{\prime}(t) \phi_{3}(t) .
\end{aligned}
$$

Substituting these values of $W_{r}$ in (9) and then (9) in (7), we get the result.

\section{Bounded solution}

In this section we obtain that all solutions of (1) are bounded. It is based on boundednees of solutions of (3) and condition (2).

Theorem 1. Let that all solutions and their derivatives up to order three of (3) be bounded in $[0, \infty)$ and the condition (2) is hold, then all the solutions of $(1)$ are bounded in $[0, \infty)$.

Proof. Let $\phi_{1}(x), \phi_{2}(x), \phi_{3}(x)$ and $\phi_{4}(x)$ be four linearly independent solutions of (3) such that $W\left(\phi_{1}, \phi_{2}, \phi_{3}, \phi_{4}\right)=1$ and let $\psi(x)$ be any solution of (1), then by Lemma 2 there are constants $c_{1}, c_{2}, c_{3}$ and $c_{4}$ such that 


$$
\begin{aligned}
\psi(x)= & c_{1} \phi_{1}(x)+c_{2} \phi_{2}(x)+c_{3} \phi_{3}(x)+c_{4} \phi_{4}(x)+\int_{0}^{x}\left[\phi_{2}(t) \phi_{3}^{\prime}(t) \phi_{4}^{\prime \prime}(t) \phi_{1}(x)\right. \\
& +\phi_{2}^{\prime \prime}(t) \phi_{3}(t) \phi_{4}^{\prime}(t) \phi_{1}(x)+\phi_{2}^{\prime}(t) \phi_{3}^{\prime \prime}(t) \phi_{4}(t) \phi_{1}(x)-\phi_{2}^{\prime}(t) \phi_{3}(t) \phi_{4}^{\prime \prime}(t) \phi_{1}(x) \\
& -\phi_{2}(t) \phi_{3}^{\prime \prime}(t) \phi_{4}^{\prime}(t) \phi_{1}(x)-\phi_{2}^{\prime \prime}(t) \phi_{3}^{\prime}(t) \phi_{4}(t) \phi_{1}(x)-\phi_{1}(t) \phi_{3}^{\prime}(t) \phi_{4}^{\prime \prime}(t) \phi_{2}(x) \\
& -\phi_{1}^{\prime \prime}(t) \phi_{3}(t) \phi_{4}^{\prime}(t) \phi_{2}(x)-\phi_{1}^{\prime}(t) \phi_{3}^{\prime \prime}(t) \phi_{4}(t) \phi_{2}(x)+\phi_{1}^{\prime}(t) \phi_{3}(t) \phi_{4}^{\prime \prime}(t) \phi_{2}(x) \\
& +\phi_{1}(t) \phi_{3}^{\prime \prime}(t) \phi_{4}^{\prime}(t) \phi_{2}(x)+\phi_{1}^{\prime \prime}(t) \phi_{3}^{\prime}(t) \phi_{4}(t) \phi_{2}(x)+\phi_{1}(t) \phi_{2}^{\prime}(t) \phi_{4}^{\prime \prime}(t) \phi_{3}(x) \\
& +\phi_{1}^{\prime \prime}(t) \phi_{2}(t) \phi_{4}^{\prime}(t) \phi_{3}(x)+\phi_{1}^{\prime}(t) \phi_{2}^{\prime \prime}(t) \phi_{4}(t) \phi_{3}(x)-\phi_{1}^{\prime}(t) \phi_{2}(t) \phi_{4}^{\prime \prime}(t) \phi_{3}(x) \\
& -\phi_{1}(t) \phi_{2}^{\prime \prime}(t) \phi_{4}^{\prime}(t) \phi_{3}(x)-\phi_{1}^{\prime \prime}(t) \phi_{2}^{\prime}(t) \phi_{4}(t) \phi_{3}(x)-\phi_{1}(t) \phi_{2}^{\prime}(t) \phi_{3}^{\prime \prime}(t) \phi_{4}(x) \\
& -\phi_{1}^{\prime \prime}(t) \phi_{2}(t) \phi_{3}^{\prime}(t) \phi_{4}(x)-\phi_{1}^{\prime}(t) \phi_{2}^{\prime \prime}(t) \phi_{3}(t) \phi_{4}(x)+\phi_{1}^{\prime}(t) \phi_{2}(t) \phi_{3}^{\prime \prime}(t) \phi_{4}(x) \\
& \left.+\phi_{1}(t) \phi_{2}^{\prime \prime}(t) \phi_{3}^{\prime}(t) \phi_{4}(x)+\phi_{1}^{\prime \prime}(t) \phi_{2}^{\prime}(t) \phi_{3}(t) \phi_{4}(x)\right] r(t) \psi(t) d t,
\end{aligned}
$$

By our hypothesis, there are constants $k_{0}, k_{1}, k_{2}$ such that $\left|\phi_{j}(x)\right| \leq k_{0},\left|\phi_{j}^{\prime}(x)\right| \leq k_{1},\left|\phi_{j}^{\prime \prime}(x)\right| \leq k_{2}$ in $[0, \infty]$.

Hence from 10 it follows that

$$
|\psi(x)| \leq\left(\left|c_{1}\right|+\left|c_{2}\right|+\left|c_{3}\right|+\left|c_{4}\right|\right) k_{0}+18 k_{0}^{2} k_{1} k_{2} \int_{0}^{x}|r(t)||\psi(t)| d t .
$$

Then, using Gronwall's Inequality, we obtain

$$
|\psi(x)| \leq\left(\left|c_{1}\right|+\left|c_{2}\right|+\left|c_{3}\right|+\left|c_{4}\right|\right) k_{0} e^{18 k_{0}^{2} k_{1} k_{2} \int_{0}^{x}|r(t)| d t} .
$$

Since by our hypothesis $\int_{0}^{x}|r(t)| d t$ is bounded in $[0, \infty)$, then $\psi(x)$ is bounded in $[0, \infty)$. which it completed the proof.

\section{$L^{4}(0, \infty)$ property of the solution}

In this section we obtain that all solutions of $(1)$ are $L^{4}(0, \infty)$ when the solutions of $(3)$ are in $L^{4}(0, \infty)$ and $r(x)$ satisfy the condition (2).

Theorem 2. Suppose that all solutions and their derivatives up to order three of (3) be in $L^{4}(0, \infty)$ and $r(x)$ is bounded in $[0, \infty)$. Then all the solutions of $(1)$ are in $L^{4}(0, \infty)$.

Proof. Let $\phi_{1}(x), \phi_{2}(x), \phi_{3}(x)$ and $\phi_{4}(x)$ be four linearly independent solutions of (3) such that $W\left(\phi_{1}, \phi_{2}, \phi_{3}, \phi_{4}\right)=1$ and let $\psi(x)$ be any solution of $(1)$, then by Lemma 2 there are constants $c_{1}, c_{2}, c_{3}$ and $c_{4}$ such that

$$
\begin{aligned}
\psi(x)= & c_{1} \phi_{1}(x)+c_{2} \phi_{2}(x)+c_{3} \phi_{3}(x)+c_{4} \phi_{4}(x)+\int_{0}^{x}\left[\phi_{2}(t) \phi_{3}^{\prime}(t) \phi_{4}^{\prime \prime}(t) \phi_{1}(x)\right. \\
& +\phi_{2}^{\prime \prime}(t) \phi_{3}(t) \phi_{4}^{\prime}(t) \phi_{1}(x)+\phi_{2}^{\prime}(t) \phi_{3}^{\prime \prime}(t) \phi_{4}(t) \phi_{1}(x)-\phi_{2}^{\prime}(t) \phi_{3}(t) \phi_{4}^{\prime \prime}(t) \phi_{1}(x) \\
& -\phi_{2}(t) \phi_{3}^{\prime \prime}(t) \phi_{4}^{\prime}(t) \phi_{1}(x)-\phi_{2}^{\prime \prime}(t) \phi_{3}^{\prime}(t) \phi_{4}(t) \phi_{1}(x)-\phi_{1}(t) \phi_{3}^{\prime}(t) \phi_{4}^{\prime \prime}(t) \phi_{2}(x) \\
& -\phi_{1}^{\prime \prime}(t) \phi_{3}(t) \phi_{4}^{\prime}(t) \phi_{2}(x)-\phi_{1}^{\prime}(t) \phi_{3}^{\prime \prime}(t) \phi_{4}(t) \phi_{2}(x)+\phi_{1}^{\prime}(t) \phi_{3}(t) \phi_{4}^{\prime \prime}(t) \phi_{2}(x) \\
& +\phi_{1}(t) \phi_{3}^{\prime \prime}(t) \phi_{4}^{\prime}(t) \phi_{2}(x)+\phi_{1}^{\prime \prime}(t) \phi_{3}^{\prime}(t) \phi_{4}(t) \phi_{2}(x)+\phi_{1}(t) \phi_{2}^{\prime}(t) \phi_{4}^{\prime \prime}(t) \phi_{3}(x) \\
& +\phi_{1}^{\prime \prime}(t) \phi_{2}(t) \phi_{4}^{\prime}(t) \phi_{3}(x)+\phi_{1}^{\prime}(t) \phi_{2}^{\prime \prime}(t) \phi_{4}(t) \phi_{3}(x)-\phi_{1}^{\prime}(t) \phi_{2}(t) \phi_{4}^{\prime \prime}(t) \phi_{3}(x) \\
& -\phi_{1}(t) \phi_{2}^{\prime \prime}(t) \phi_{4}^{\prime}(t) \phi_{3}(x)-\phi_{1}^{\prime \prime}(t) \phi_{2}^{\prime}(t) \phi_{4}(t) \phi_{3}(x)-\phi_{1}(t) \phi_{2}^{\prime}(t) \phi_{3}^{\prime \prime}(t) \phi_{4}(x) \\
& -\phi_{1}^{\prime \prime}(t) \phi_{2}(t) \phi_{3}^{\prime}(t) \phi_{4}(x)-\phi_{1}^{\prime}(t) \phi_{2}^{\prime \prime}(t) \phi_{3}(t) \phi_{4}(x)+\phi_{1}^{\prime}(t) \phi_{2}(t) \phi_{3}^{\prime \prime}(t) \phi_{4}(x) \\
& \left.+\phi_{1}(t) \phi_{2}^{\prime \prime}(t) \phi_{3}^{\prime}(t) \phi_{4}(x)+\phi_{1}^{\prime \prime}(t) \phi_{2}^{\prime}(t) \phi_{3}(t) \phi_{4}(x)\right] r(t) \psi(t) d t .
\end{aligned}
$$


Then by hypothesis there are constants $C, k_{0}, k_{1}, k_{2}$ such that $|r(x)| \leq C$ in $[0, \infty)$, and $\int_{0}^{\infty}\left|\phi_{j}(x)\right|^{4} d x \leq k_{0}, \quad \int_{0}^{\infty}\left|\phi_{j}^{\prime}(x)\right|^{4} d x \leq k_{1}, \quad \int_{0}^{\infty}\left|\phi_{j}^{\prime \prime}(x)\right|^{4} d x \leq k_{2}$ for $j=1,2,3,4$.

Now, applying the Holder's inequality for integral, we get

$$
\begin{aligned}
& \mid \int_{0}^{x}\left[\phi_{2}(t) \phi_{3}^{\prime}(t) \phi_{4}^{\prime \prime}(t) \phi_{1}(x)+\phi_{2}^{\prime \prime}(t) \phi_{3}(t) \phi_{4}^{\prime}(t) \phi_{1}(x)+\phi_{2}^{\prime}(t) \phi_{3}^{\prime \prime}(t) \phi_{4}(t) \phi_{1}(x)\right. \\
& -\phi_{2}^{\prime}(t) \phi_{3}(t) \phi_{4}^{\prime \prime}(t) \phi_{1}(x)-\phi_{2}(t) \phi_{3}^{\prime \prime}(t) \phi_{4}^{\prime}(t) \phi_{1}(x)-\phi_{2}^{\prime \prime}(t) \phi_{3}^{\prime}(t) \phi_{4}(t) \phi_{1}(x) \\
& -\phi_{1}(t) \phi_{3}^{\prime}(t) \phi_{4}^{\prime \prime}(t) \phi_{2}(x)-\phi_{1}^{\prime \prime}(t) \phi_{3}(t) \phi_{4}^{\prime}(t) \phi_{2}(x)-\phi_{1}^{\prime}(t) \phi_{3}^{\prime \prime}(t) \phi_{4}(t) \phi_{2}(x) \\
& +\phi_{1}^{\prime}(t) \phi_{3}(t) \phi_{4}^{\prime \prime}(t) \phi_{2}(x)+\phi_{1}(t) \phi_{3}^{\prime \prime}(t) \phi_{4}^{\prime}(t) \phi_{2}(x)+\phi_{1}^{\prime \prime}(t) \phi_{3}^{\prime}(t) \phi_{4}(t) \phi_{2}(x) \\
& +\phi_{1}(t) \phi_{2}^{\prime}(t) \phi_{4}^{\prime \prime}(t) \phi_{3}(x)+\phi_{1}^{\prime \prime}(t) \phi_{2}(t) \phi_{4}^{\prime}(t) \phi_{3}(x)+\phi_{1}^{\prime}(t) \phi_{2}^{\prime \prime}(t) \phi_{4}(t) \phi_{3}(x) \\
& -\phi_{1}^{\prime}(t) \phi_{2}(t) \phi_{4}^{\prime \prime}(t) \phi_{3}(x)-\phi_{1}(t) \phi_{2}^{\prime \prime}(t) \phi_{4}^{\prime}(t) \phi_{3}(x)-\phi_{1}^{\prime \prime}(t) \phi_{2}^{\prime}(t) \phi_{4}(t) \phi_{3}(x) \\
& -\phi_{1}(t) \phi_{2}^{\prime}(t) \phi_{3}^{\prime \prime}(t) \phi_{4}(x)-\phi_{1}^{\prime \prime}(t) \phi_{2}(t) \phi_{3}^{\prime}(t) \phi_{4}(x)-\phi_{1}^{\prime}(t) \phi_{2}^{\prime \prime}(t) \phi_{3}(t) \phi_{4}(x) \\
& \left.+\phi_{1}^{\prime}(t) \phi_{2}(t) \phi_{3}^{\prime \prime}(t) \phi_{4}(x)+\phi_{1}(t) \phi_{2}^{\prime \prime}(t) \phi_{3}^{\prime}(t) \phi_{4}(x)+\phi_{1}^{\prime \prime}(t) \phi_{2}^{\prime}(t) \phi_{3}(t) \phi_{4}(x)\right] \\
& \times r(t) \psi(t) d t \mid \\
\leq & 6 C\left|\phi_{1}(x)\right|\left(k_{0} k_{1} k_{2}\right)^{\frac{1}{4}}\left[\int_{0}^{x}|\psi(t)|^{4} d t\right]^{\frac{1}{4}}+6 C\left|\phi_{2}(x)\right|\left(k_{0} k_{1} k_{2}\right)^{\frac{1}{4}}\left[\int_{0}^{x}|\psi(t)|^{4} d t\right]^{\frac{1}{4}} \\
& +6 C\left|\phi_{3}(x)\right|\left(k_{0} k_{1} k_{2}\right)^{\frac{1}{4}}\left[\int_{0}^{x}|\psi(t)|^{4} d t\right]^{\frac{1}{4}}+6 C\left|\phi_{4}(x)\right|\left(k_{0} k_{1} k_{2}\right)^{\frac{1}{4}}\left[\int_{0}^{x}|\psi(t)|^{4} d t\right]^{\frac{1}{4}} \\
= & 6 C\left(k_{0} k_{1} k_{2}\right)^{\frac{1}{4}}\left(\left|\phi_{1}(x)\right|+\left|\phi_{2}(x)\right|+\left|\phi_{3}(x)\right|+\left|\phi_{4}(x)\right|\right) \Psi^{\frac{1}{4}}(x) .
\end{aligned}
$$

Now, from the equation (11) it follows that

$$
\begin{aligned}
& |\psi(x)| \leq\left|c_{1}\right|\left|\phi_{1}(x)\right|+\left|c_{2}\right|\left|\phi_{2}(x)\right|+\left|c_{3}\right|\left|\phi_{3}(x)\right|+\left|c_{4}\right|\left|\phi_{4}(x)\right| \\
& +6 C\left(k_{0} k_{1} k_{2}\right)^{\frac{1}{4}}\left(\left|\phi_{1}(x)\right|+\left|\phi_{2}(x)\right|+\left|\phi_{3}(x)\right|+\left|\phi_{4}(x)\right|\right) \Psi^{\frac{1}{4}}(x) .
\end{aligned}
$$

Then

$$
\begin{aligned}
|\psi(x)|^{4} \leq & \left(\left|c_{1}\right|\left|\phi_{1}(x)\right|+\left|c_{2}\right|\left|\phi_{2}(x)\right|+\left|c_{3}\right|\left|\phi_{3}(x)\right|+\left|c_{4}\right|\left|\phi_{4}(x)\right|\right. \\
& \left.+6 C\left(k_{0} k_{1} k_{2}\right)^{\frac{1}{4}}\left(\left|\phi_{1}(x)\right|+\left|\phi_{2}(x)\right|+\left|\phi_{3}(x)\right|+\left|\phi_{4}(x)\right|\right) \Psi^{\frac{1}{4}}(x)\right)^{4} .
\end{aligned}
$$

Using the elementary inequality for any two real numbers $x, y$

$$
(x+y)^{4} \leq 8\left(x^{4}+y^{4}\right)
$$

and equation (12), we get

$$
\begin{aligned}
|\psi(x)|^{4} \leq & \left(\left|c_{1}\right|\left|\phi_{1}(x)\right|+\left|c_{2}\right|\left|\phi_{2}(x)\right|+\left|c_{3}\right|\left|\phi_{3}(x)\right|+\left|c_{4}\right|\left|\phi_{4}(x)\right|\right)^{4} \\
& +1296 C^{4}\left(k_{0} k_{1} k_{2}\right)\left(\left|\phi_{1}(x)\right|+\left|\phi_{2}(x)\right|+\left|\phi_{3}(x)\right|+\left|\phi_{4}(x)\right|\right)^{4} \Psi(x) .
\end{aligned}
$$

Using the elementary inequality for any real numbers $a, b, c, d$

$$
(a+b+c+d)^{4} \leq 64\left(a^{4}+b^{4}+c^{4}+d^{4}\right)
$$

and equation (13), we obtain

$$
\begin{aligned}
|\psi(x)|^{4} \leq & 64\left(\left|c_{1}\right|^{4}\left|\phi_{1}(x)\right|^{4}+\left|c_{2}\right|^{4}\left|\phi_{2}(x)\right|^{4}+\left|c_{3}\right|^{4}\left|\phi_{3}(x)\right|^{4}+\left|c_{4}\right|^{4}\left|\phi_{4}(x)\right|^{4}\right) \\
& +82944 C^{4}\left(k_{0} k_{1} k_{2}\right)\left(\left|\phi_{1}(x)\right|^{4}+\left|\phi_{2}(x)\right|^{4}+\left|\phi_{3}(x)\right|^{4}+\left|\phi_{4}(x)\right|^{4}\right) \\
& \times \Psi(x) .
\end{aligned}
$$


Integrating (14) over $[0, X]$, we can write

$$
\begin{aligned}
\int_{0}^{X}|\psi(x)|^{4} d x \leq & 64\left(\left|c_{1}\right|^{4} \int_{0}^{X}\left|\phi_{1}(x)\right|^{4} d x+\left|c_{2}\right|^{4} \int_{0}^{X}\left|\phi_{2}(x)\right|^{4} d x\right. \\
& \left.+\left|c_{3}\right|^{4} \int_{0}^{X}\left|\phi_{3}(x)\right|^{4} d x+\left|c_{4}\right|^{4} \int_{0}^{X}\left|\phi_{4}(x)\right|^{4} d x\right) \\
& +82944 C^{4}\left(k_{0} k_{1} k_{2}\right) \int_{0}^{X}\left(\left|\phi_{1}(x)\right|^{4}+\left|\phi_{2}(x)\right|^{4}\right. \\
& \left.+\left|\phi_{3}(x)\right|^{4}+\left|\phi_{4}(x)\right|^{4}\right) \Psi(x) d x \\
\leq & 64 k_{0}\left(\left|c_{1}\right|^{4}+\left|c_{2}\right|^{4}+\left|c_{3}\right|^{4}+\left|c_{4}\right|^{4}\right) \\
& +82944 C^{4}\left(k_{0} k_{1} k_{2}\right) \int_{0}^{X}\left(\left|\phi_{1}(x)\right|^{4}+\left|\phi_{2}(x)\right|^{4}\right. \\
& \left.+\left|\phi_{3}(x)\right|^{4}+\left|\phi_{4}(x)\right|^{4}\right) \Psi(x) d x .
\end{aligned}
$$

That means

$$
\begin{aligned}
& \Psi(x) \leq 64 k_{0}\left(\left|c_{1}\right|^{4}+\left|c_{2}\right|^{4}+\left|c_{3}\right|^{4}+\left|c_{4}\right|^{4}\right) \\
& +82944 C^{4}\left(k_{0} k_{1} k_{2}\right) \int_{0}^{X}\left(\left|\phi_{1}(x)\right|^{4}+\left|\phi_{2}(x)\right|^{4}+\left|\phi_{3}(x)\right|^{4}+\left|\phi_{4}(x)\right|^{4}\right) \Psi(x) d x .
\end{aligned}
$$

Then, using the Gronwall's Inequality, we obtain

$$
\begin{aligned}
\Psi(X) \leq & 64 k_{0}\left(\left|c_{1}\right|^{4}+\left|c_{2}\right|^{4}+\left|c_{3}\right|^{4}+\left|c_{4}\right|^{4}\right) \\
& \times e^{82944 C^{4}\left(k_{0} k_{1} k_{2}\right) \int_{0}^{X}\left(\left|\phi_{1}(x)\right|^{4}+\left|\phi_{2}(x)\right|^{4}+\left|\phi_{3}(x)\right|^{4}+\left|\phi_{4}(x)\right|^{4}\right) d x} .
\end{aligned}
$$

This means that $\Psi(x)$ is a bounded as $X \rightarrow \infty$. Thus we get $\psi(x) \in L^{4}(0, \infty)$.

Corollary 1. Let $\lambda$ be a complex parameter and there be a value $\lambda_{0}$ such that all solution and their derivatives up to order three of the equation

$$
y^{(4)}+\{\lambda-Q(x)\} y(x)=0
$$

are in $L^{4}(0, \infty)$ when $\lambda=\lambda_{0}$. Then all solutions of the equation are in $L^{4}(0, \infty)$ for every $\lambda$.

Proof. We can write

$$
\lambda-Q(x)=\lambda_{0}+Q(x)+\left(\lambda-\lambda_{0}\right) .
$$

Then the differential equation has the following form

$$
y^{(4)}+\left\{\lambda_{0}-Q(x)+\left(\lambda-\lambda_{0}\right)\right\} y(x)=0 .
$$

Comparing with (1) and (3), we obtain $q(x)=\lambda_{0}-Q(x)$ and $r(x)=\lambda-\lambda_{0}$. This means that $r(x)$ is a constant function which is bounded in $[0, \infty)$. Then by using the Theorem 3 we obtain that all solutions of $(15)$ are in $L^{4}(0, \infty)$ for every $\lambda$.

\section{Conclusion}

In the present paper, we study some properties of a general linear differential equation of fourth order in infinite interval of the form: $y^{(4)}(x)+\{q(x)+r(x)\} y(x)=0, \quad 0 \leq x<\infty$, where $r(x)$ is a function which satisfies the condition: $\int_{0}^{\infty}|r(x)| d x<\infty$. A simple application of this result is provided. 


\section{References}

1 Jwamer K.H. Estimates for the eigenfunctions of the Regge Problem / K.H. Jwamer, G. Aigunov // Mathematical Notes - 2012. - 92. - No. 7. - P. 127-130.

2 Eastham M.S.P. Theory of ordinary differential equations / M.S.P. Eastham // Van Nostrand Reinhold London, Florence, Kentucky, U.S.A. 1970.

3 Aigunov G.A. Estimation of normalized eigenfunctions of problem of T.Regge type in case of smooth coefficients / G.A. Aigunov, T.Y. Gadzhieva // Funct. Differ. equations their Appl. 2009. - No. 5.

4 Aigunov G.A. Estimation of normalized eigenfunctions of problem of T.Regge type in case of smooth coefficients / G.A. Aigounov, Y. Tamila

Interuniv. Res.-themed Collect. Funct. equations their Appl. Makhachkala (South Russ.) - 2009. - No. 5. - P. 18-26.

5 Sargsjan I.S. Introduction to spectral theory: selfadjoint ordinary differential operators / I.S. Sargsjan, B.M. Levitan. - Rhode Island: American, 1975.

6 Mamedov K. R. On the Riesz Basis Property of the Root Functions in Certain Regular Boundary Value Problems / K. Mamedov, N.B. Kerimov // Kluwer Academic/Plenum Publishers, 65, 1998.

7 Kerimov N.B. On the Riesz Basis Property of the Root Functions in Certain Regular Boundary Value Problems / N.B. Kerimov, K.R. Mamedov // Matemalicheskie zametki. - 1999. - 65. No. 4. - P. 483-487.

8 Kerimov N.B. Some problems of spectral theory of fourth-order differential operators with regular boundary conditions / N.B. Kerimov, U.Kaya. - Springer, 2013.

9 Jwamer K.H. Estimations of the upper bound for the eigen-functions of the fourth order boundary value problem with smooth coefficients / K.H. Jwamer, R.R. Qadir // Math. Sci. Lett. - 2017. - 6. - No. 1. - P. 67-74.

10 Qadir R.R. Refinement Asymptotic Formulas of Eigenvalues and Eigenfunctions of a Fourth Order Linear Differential Operator with Transmission Condition and Discontinuous Weight Function / R.R. Qadir, K.H.F. Jwamer // Symmetry - 2019. - 11. - No. 8. - P. 1060.

11 Jwamer K.H.F. Estimation of normalized eigenfunctions of spectral problem with smooth coefficients / K.H.F. Jwamer, R.R. Qadir // Journal of Chemical Information and Modeling, - 2013. - 53. - No. 9. - P. 1689-1699.

Карван Х.Ф. Жвемер, Рандо Р.К. Расул

\section{Төртінші ретті сызықты дифференциалдық теңдеуді оның шеттік есебімен салыстыру}

\footnotetext{
Мақалада төртінші ретті сызықты дифференциалдық теңдеу қарастырылған. Авторлар бұл дифференциалдық теңдеудің жоғарғы бағамын, сонымен қатар барлық шешімі $L^{4}(0, \infty)$ табылатындығын дәлелдеген. Алынған нәтижелерді салыстыра келе, осы дифференциалдық теңдеуден туындаған шеттік есептің барлық меншікті функциялары шектелген және $L^{4}(0, \infty)$ орналасқан болып табылады.

Kiлm сөздер: сызықты дифференциалдық теңдеу, меншікті мән, меншікті функция, жоғарғы бағамы, сызықты тәуелсіз шешімі, $L^{2}(0$, infty), вронскиан, Гронуолла теңсіздігі, тұрақтыны варияциялау.
} 
Карван Х.Ф. Жвемер, Рандо Р.К. Расул

\title{
Сравнение линейного дифференциального уравнения четвертого порядка с его краевой задачей
}

\begin{abstract}
В статье изучено линейное дифференциальное уравнение четвертого порядка. Авторами найдена верхняя оценка для решений этого дифференциального уравнения, а также доказано, что все решения находятся в $L^{4}(0, \infty)$. Сравнивая эти результаты, авторы пришли к выводу, что все собственные функции краевой задачи, порожденные этим дифференциальным уравнением, ограничены и находятся в $L^{4}(0, \infty)$.

Ключевые слова: линейное дифференциальное уравнение, собственное значение, собственная функция, верхняя оценка, линейно независимое решение, $L^{2}(0$, infty), вронскиан, неравенство Гронуолла, вариация постоянных (параметров).
\end{abstract}

\section{References}

1 Jwamer, K.H., \& Aigunov, G. (2012). Estimates for the eigenfunctions of the Regge Problem. Mathematical Notes, 92, 7, 127-130.

2 Eastham, M.S.P. (1970). Theory of ordinary differential equations. Van Nostrand Reinhold London, Florence, Kentucky, U.S.A.

3 Aigunov, G.A., \& Gadzhieva, T.Y. (2009). Estimation of normalized eigenfunctions of problem of T. Regge type in case of smooth coefficients. Funct. Differ. equations their Appl. 5.

4 Aigunov, G.A., \& Tamila, Y. (2009). Estimation of normalized eigenfunctions of problem of T.Regge type in case of smooth coefficients. Interuniv. Res.-themed Collect. Funct. equations their Appl. Makhachkala (South Russ.), 5, 18-26.

5 Sargsjan, I.S., \& Levitan, B.M. (1975). Introduction to spectral theory: selfadjoint ordinary differential operators. Rhode Island: American, 1975.

6 Mamedov, K.R., \& Kerimov, N.B. (1998). On the Riesz Basis Property of the Root Functions in Certain Regular Boundary Value Problems. Kluwer Academic/Plenum Publishers, 65, 1998.

7 Kerimov, N.B., \& Mamedov,K.R. (1999). On the Riesz Basis Property of the Root Functions in Certain Regular Boundary Value Problems. Matemalicheskie Zametki, 65, 4, 483-487.

8 Kerimov, N.B., \& Kaya, U. (2013). Some problems of spectral theory of fourth-order differential operators with regular boundary conditions, Springer.

9 Jwamer, K.H., \& Qadir, R.R. (2017). Estimations of the upper bound for the eigen-functions of the fourth order boundary value problem with smooth coefficients. Math. Sci. Lett., 6, 1, 67-74.

10 Qadir, R.R., \& Jwamer, K.H.F. (2019). Refinement Asymptotic Formulas of Eigenvalues and Eigenfunctions of a Fourth Order Linear Differential Operator with Transmission Condition and Discontinuous Weight Function. Symmetry, 11, 8, 1060.

11 Jwamer, K.H.F., \& Qadir, R.R. (2013). Estimation of normalized eigenfunctions of spectral problem with smooth coefficients. Journal of Chemical Information and Modeling, 53, 9, 16891699. 